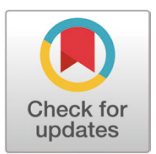

Received: May 27, 2020

Revised: Jul 1, 2020

Accepted: Jul 7, 2020

*Corresponding author

Wan-Kyu Lee

Laboratory of Veterinary Bacteriology

and Infectious Diseases,

College of Veterinary Medicine,

Chungbuk National University,

Cheongju 28644, Korea.

Tel: +82-43-261-2960

E-mail: wklee@cbu.ac.kr

Copyright $\odot 2020$ Korean Society of Animal Sciences and Technology.

This is an Open Access article distributed under the terms of the Creative Commons Attribution Non-Commercial License (http:// creativecommons.org/licenses/by$\mathrm{nc} / 4.0 /$ ) which permits unrestricted non-commercial use, distribution, and reproduction in any medium, provided the original work is properly cited.

ORCID

Kyung-Hyo Do

https://orcid.org/0000-0001-6080-0374 Jae-Won Byun

https://orcid.org/0000-0001-5664-2107

Wan-Kyu Lee

https://orcid.org/0000-0001-5087-6359

Competing interests

No potential conflict of interest relevant to this article was reported.

Funding sources

This work was supported by "Korea Institute of Planning and Evaluation for Technology in Food, Agriculture,

Forestry and Fisheries(IPET) through Agriculture, Food and Rural Affairs

\section{Virulence genes and antimicrobial resistance of pathogenic Escherichia coli isolated from diarrheic weaned piglets in Korea}

\author{
Kyung-Hyo Do ${ }^{1}$, Jae-Won Byun ${ }^{2}$ and Wan-Kyu Lee ${ }^{1 *}$ \\ ${ }^{1}$ Laboratory of Veterinary Bacteriology and Infectious Diseases, College of Veterinary Medicine, \\ Chungbuk National University, Cheongju 28644, Korea \\ ${ }^{2}$ Animal Disease Diagnostic Division, Animal and Plant Quarantine Agency, Gimcheon 39660, Korea
}

\begin{abstract}
For efficient prevention and treatment of enteric colibacillosis, understanding about latest virulence factors and antimicrobial resistance of Escherichia coli is essentially needed. The aim of this study was to survey antimicrobial resistance and determine the prevalence of fimbriae and enterotoxin genes among 118 pathogenic $E$. coli isolates obtained from Korean pigs with diarrhea between 2016 and 2017. The genes for the toxins and adhesins were amplified by polymerase chain reaction (PCR). The susceptibility of the $E$. coli isolates to antimicrobials were tested using the standard Kirby-Bauer disk diffusion method. The most prevalent fimbrial antigen was F18 (40.7\%), followed by F4 (16.9\%), and the most prevalent combinations of toxin genes were Stx2e (21.2\%), STb:EAST-1 (19.5\%), and STa:STb (16.9\%), respectively. Among the pathotypes, enterotoxigenic E. coli (ETEC) was the most predominant (67.8\%), followed by Shiga-toxin producing E. coli (STEC, 23.7\%). We confirmed high resistance rates to chloramphenicol $(88.1 \%)$, tetracycline $(86.4 \%)$, streptomycin $(86.4 \%)$, and ampicillin (86.4\%). And the majorities of isolates $(90.7 \%)$ showed multi-drug resistance which means having resistance to 3 or more subclasses of antimicrobials. Results of this study can be a source of valuable data for investigating the epidemiology of and control measures for enteric colibacillosis in Korean piggeries.
\end{abstract}

Keywords: Escherichia coli, Piglets, Colibacillosis, Antimicrobial resistance, Virulence profiles

\section{INTRODUCTION}

Escherichia coli causes enteric colibacillosis, a common disease of nursing and weanling pigs that can result in neonatal diarrhea, post-weaning diarrhea, and edema disease. This disease has a significant economic impact on the pig industry due to its association with a high morbidity and mortality, as well as a decreased weight gain in pigs [1].

Enterotoxigenic $E$. coli (ETEC) causes diarrhea by inducing the production of heat labile (LT) and heat stable (ST) toxins (STa and $\mathrm{STb}$ ), as well as an enteroaggregative $E$. coli heat-stable enterotoxin-1 (EAST-1). Furthermore, the Shiga-toxin producing E. coli (STEC) causes edema disease via the pro- 
Convergence Technologies Program for Educating Creative Global Leader, funded by Ministry of Agriculture, Food and Rural Affairs(MAFRA)(grant number: 320005-4)".

\section{Acknowledgements}

Not applicable.

\section{Availability of data and material Upon reasonable request, the datasets of this study can be available from the corresponding author.}

Authors' contributions

Conceptualization: Do KH, Byun JW, Lee WK.

Data Curation: Do KH.

Formal analysis: Do KH, Byun JW.

Methodology: Byun JW.

Validation: Do KH.

Investigation: Do KH.

Resources: Byun JW, Lee WK.

Writing-original draft preparation: Do KH.

Writing-review and editing: Do KH, Byun JW, Lee WK.

Ethics approval and consent to participate This article does not require IRB/IACUC approval because there are no human and animal participants. duction of the Shiga-toxin variant, Stx2e [2]

Pathogenic $E$. coli produce enterotoxins by attaching itself to intestinal epithelial cells. Fimbriae and non-fimbrial adhesins play a significant role in the adhesion of these bacteria to intestinal mucosa and epithelial cells [3]. Since the pathogenicity of $E$. coli is determined depending on virulence factors, the identification of the virulence factors of $E$. coli is key for the diagnosis of swine enteric colibacillosis [1].

Antibiotics have played a significant role in the prevention and treatment of diseases in the pig industry. The Korean swine industry has used them for the prevention of diseases and the promotion of growth for many years in the form of feed additives [4]. However, the indiscriminate use of antibiotics has resulted in the emergence of antimicrobial-resistant bacteria and the appearance of antibiotic residues in meat products. As such, antibiotic growth promoters were banned in Korea in July 2011 [5].

Due to the emergence of antimicrobial-resistant bacteria and the spread of antimicrobial resistance genes, antimicrobial resistance has become an international problem. To overcome this problem, Denmark [6],Japan [7], and Canada [8] have started to formally monitor the state of antibiotic resistance. The frequency of virulence factors and antimicrobial resistance patterns of pathogenic E. coli is known to vary over time and region [9]. To devise control measures for colibacillosis in piggeries, data regarding the prevalence of virulence factors and antimicrobial susceptibility of $E$. coli are needed. Therefore, to improve the productivity of the Korean domestic pig industry, the monitoring of antimicrobial resistance patterns and virulence factors of pathogenic $E$. coli in Korean pig farms is needed [10]. The aim of this study was to survey antimicrobial resistance and determine the prevalence of fimbriae and enterotoxin genes among 118 pathogenic $E$. coli isolates obtained from Korean pigs with diarrhea between 2016 and 2017.

\section{MATERIALS AND METHODS}

\section{E. coli isolates}

Between 2016 and 2017, 118 E. coli isolates were obtained from weaned piglets showing symptoms of diarrhea or edema disease. The sampled farms consisted of 72 different pig herds (50 to 100 sows per each herd) and were located in three areas: northern (21 farms encompassing the Gangwon, Gyeonggi, and Incheon provinces), central (27 farms, Chungbuk and Chungnam provinces), and southern (24 farms, Chonbuk, Chonnam, Gyeongbuk, and Gyeongnam provinces) Korea. Strains were not collected repeatedly from the same farm. Intestinal and fecal samples were collected using sterilized swab from weaned piglets showing diarrhea and/or sudden death with neurological symptoms. The aseptically collected intestinal and fecal samples were inoculated onto MacConkey agar (Becton Dickinson, MD, USA). After overnight incubation at $37^{\circ} \mathrm{C}$, only pure cultured colonies pink in color were selected and transferred to blood agar (Asan Pharmaceutical, Korea). Suspected colonies were identified as E. coli by using the VITEK II system (bioMéreiux, Marcy I'Etoile, France). $\alpha$ - and $\beta$-hemolysis was also determined in blood agar. The tested isolates were stored in $50 \%$ glycerol stock at $-70^{\circ} \mathrm{C}$ until further characterization.

\section{Determination of virulence genes}

The E. coli gene for the toxins (LT, STa, STb, Stx2e, and EAST-1), fimbriae (F4, F5, F6, F18, and F41), and non-fimbrial adhesins (AIDA-1, paa, eae) were amplified by polymerase chain reaction (PCR), which was performed using previously described protocols [9]. All primers used in the study are listed in Table 1. Bacterial colonies were suspended in distilled water and boiled for $10 \mathrm{~min}$. After centrifugation at $8,000 \times \mathrm{g}$, the supernatant was used as a template for PCR. The reaction volume 
Table 1. Primers used to detect colonization factors of Escherichia coli from diarrheic weaned piglets in Korea

\begin{tabular}{|c|c|c|}
\hline Primer & Oligonucleotide sequence & Product size (bp) \\
\hline \multirow[t]{2}{*}{ F4 } & TGAATGACCTGACCAATGGTGGAACC & 484 \\
\hline & GCGTTTACTCTTTGAATCTGTCCGAG & \\
\hline \multirow[t]{2}{*}{ F5 } & GCGACTACCAATGCTTCTGCGAATAC & 230 \\
\hline & GAACCAGACCAGTCAATACGAGCA & \\
\hline \multirow[t]{2}{*}{ F6 } & GCCAGTCTATGCCAAGTGGATACTTC & 391 \\
\hline & GTTTGTATCAGGATTCCCTGTGGTGG & \\
\hline \multirow[t]{2}{*}{ F18 } & TGGCACTGTAGGAGATACCATTCAGC & 230 \\
\hline & GGTTTGACCACCTTTCAGTTGAGCAG & \\
\hline \multirow[t]{2}{*}{ F41 } & TTAGCAGCGAAGATGAGTGATGGG & 515 \\
\hline & GTACTACCTGCAGAAACACCAGATCC & \\
\hline \multirow[t]{2}{*}{ eae } & CATTATGGAACGGCAGAGGT & 791 \\
\hline & АTCTTCTGCGTACTGCGTTCA & \\
\hline \multirow[t]{2}{*}{ paa } & TGGCTGGACCAGGAAAGGCACT & 584 \\
\hline & AAGTGCGGGTGCGTTGAGGATG & \\
\hline \multirow[t]{2}{*}{ AIDA-I } & AGTGGCGGGGCTCAGAACATCT & 273 \\
\hline & CTCAGTGGCATTAGCGCCAGCA & \\
\hline \multirow[t]{2}{*}{ LT } & ACGGCGTTACTATCCTGTCTATGTGC & 275 \\
\hline & TTGGTCTCGGTCAGATATGTGATTC & \\
\hline \multirow[t]{2}{*}{ STa } & GTCAGTCAACTGAATCACTTGACTCT & 152 \\
\hline & CATGGAGCACAGGCAGGATTACAACA & \\
\hline \multirow[t]{2}{*}{$\mathrm{STb}$} & GCTACAAATGCCTATGCATCTACACA & 125 \\
\hline & CATGCTCCAGCAGTACCATCTCTAAC & \\
\hline \multirow[t]{2}{*}{ Stx2e } & CGGTATCCTATTCCCAGGAGTTTACG & 599 \\
\hline & GTCTTCCGGCGTCATCGTATAAACAG & \\
\hline \multirow[t]{2}{*}{ EAST-I } & CTGGCCGAAAATGAAGGGGCGA & 125 \\
\hline & TAACTGGATGCGGGCCTTCGGA & \\
\hline
\end{tabular}

$(20 \mu \mathrm{L})$ was composed of $2 \times$ emeraldAmp Master Mix (Takara, Japan), $2 \mu \mathrm{M}$ of each primer, and $3 \mu \mathrm{L}$ of DNA template. After amplification, the products were visualized by electrophoresis in $2 \%$ agarose gels stained with ethidium bromide.

\section{Antimicrobial resistance}

Antimicrobial susceptibility testing was carried out using the Kirby-Bauer disk diffusion method [11]. The following 16 antimicrobials were selected after referring to the Clinical and Laboratory Standards Institute (CLSI) guidance [12]: gentamicin (10 $\mu \mathrm{g})$, streptomycin $(10 \mu \mathrm{g})$, neomycin (30 $\mu \mathrm{g})$, cephalothin $(30 \mu \mathrm{g})$, cefazolin $(30 \mu \mathrm{g})$, cefepime $(30 \mu \mathrm{g})$, cefoxitin $(30 \mu \mathrm{g})$, nalidixic acid $(30$ $\mu \mathrm{g})$, ciprofloxacin $(5 \mu \mathrm{g})$, norfloxacin $(10 \mu \mathrm{g})$, ampicillin $(10 \mu \mathrm{g})$, amoxicillin/clavulanic acid (20/10 $\mu \mathrm{g})$, trimethoprim/sulfamethoxazole (23.75/1.25 $\mu \mathrm{g})$, chloramphenicol (30 $\mu \mathrm{g})$, colistin $(10 \mu \mathrm{g})$, and tetracycline $(30 \mu \mathrm{g})$. Each antimicrobial disc was purchased from Becton-Dickinson (Becton Dickinson, MD, USA). Strains resistant to three or more CLSI subclass of drugs according to Magiorakos criteria were considered as multi-drug resistant strains [13]. 


\section{RESULTS}

\section{Frequency of virotypes (combination of toxin and fimbriae)}

The frequency of the virotypes of pathogenic $E$. coli isolated from Korean diarrheic pigs is shown in Table 2. The most prevalent fimbrial antigen was F18 (48 of 118), followed by F4 (20 of 118). On the other hand, only 3 isolates were determined as being F5-positive, 2 isolates were determined as F18 with F4, and no isolates were determined as F41. Forty-four isolates did not contain any fimbrial antigens. The most prevalent combinations of toxin genes were Stx2e (25 isolates), STb:EAST-1 (23 isolates), and $\mathrm{STa}: \mathrm{STb}$ (20 isolates), respectively. Among these, STb:EAST-1 without fimbrial gene was the most prevalent virotype (22 isolates), followed by F18:Stx2e (15 isolates), F18:STa:STb (12 isolates), and F4:LT:STb:EAST-1 (11 isolates), respectively.

\section{Association between fimbrial and non-fimbrial adhesin genes}

Table 3 shows the relationship between fimbrial and non-fimbrial adhesin genes. Among 118 isolates, 39 and 26 were AIDA- 1 and paa-positive, respectively. On the other hand, only 1 isolate was eae-positive. All the paa- and eae-positive isolates and 97.4\% (38 of 39) of the AIDA-1- positive

Table 2. Relationship between fimbriae and enterotoxins in 118 Escherichia coli isolates obtained from pigs with diarrhea in Korea

\begin{tabular}{|c|c|c|c|c|c|c|c|}
\hline \multirow{2}{*}{ Enterotoxins } & \multicolumn{7}{|c|}{ Fimbriae } \\
\hline & F4 & F5 & F18 & F4:F6 & F4:F18 & None & Total \\
\hline LT & 0 & 0 & 1 & 0 & 0 & 0 & 1 \\
\hline STa & 0 & 1 & 1 & 0 & 0 & 1 & 3 \\
\hline STb & 2 & 0 & 0 & 0 & 0 & 3 & 5 \\
\hline EAST1 & 1 & 0 & 2 & 0 & 0 & 2 & 5 \\
\hline Stx2e & 1 & 0 & 15 & 0 & 0 & 9 & 25 \\
\hline STa:STb & 4 & 1 & 12 & 1 & 0 & 2 & 20 \\
\hline STa:EAST-1 & 0 & 0 & 2 & 0 & 0 & 0 & 2 \\
\hline STb:EAST-1 & 0 & 0 & 1 & 0 & 0 & 22 & 23 \\
\hline STa:Stx2e & 0 & 0 & 4 & 0 & 0 & 0 & 4 \\
\hline Stx2e:EAST-1 & 0 & 0 & 4 & 0 & 0 & 2 & 6 \\
\hline LT:STb:EAST-1 & 11 & 0 & 1 & 0 & 1 & 2 & 15 \\
\hline STa:STb:EAST-1 & 1 & 0 & 0 & 0 & 0 & 0 & 1 \\
\hline LT:Stx2e:EAST-1 & 0 & 0 & 1 & 0 & 0 & 0 & 1 \\
\hline STa:STb:Stx2e & 0 & 0 & 1 & 0 & 0 & 1 & 2 \\
\hline LT:STa:STb:EAST-1 & 0 & 0 & 2 & 0 & 1 & 0 & 3 \\
\hline None & 0 & 1 & 1 & 0 & 0 & 0 & 2 \\
\hline Total & 20 & 3 & 48 & 1 & 2 & 44 & 118 \\
\hline
\end{tabular}

Table 3. Relationship between fimbrial and non-fimbrial adhesins

\begin{tabular}{lrcc}
\hline & \multicolumn{3}{c}{ Non-fimbrial adhesins (\%) } \\
\cline { 2 - 4 } & $\begin{array}{c}\text { AlDA-1 } \\
(\mathbf{n}=\mathbf{3 9})\end{array}$ & $\begin{array}{c}\text { paa } \\
(\mathbf{n = 2 6 )}\end{array}$ & $\begin{array}{c}\text { eae } \\
(\mathbf{n}=\mathbf{1})\end{array}$ \\
\hline F4 $(\mathrm{n}=23)$ & $1(2.6)$ & $6(23.1)$ & $0(0.0)$ \\
F18 $(\mathrm{n}=50)$ & $19(48.7)$ & $10(38.5)$ & $1(100.0)$ \\
Toxin genes ${ }^{1)}$ & $38(97.4)$ & $26(100.0)$ & $1(100.0)$ \\
\hline
\end{tabular}

${ }^{11}$ Isolates with at least one enterotoxin gene, including LT, STa, STb, Stx2e, and EAST-1. 
isolates contained enterotoxin genes (LT, STa, STb, Stx2e, and EAST-1). Furthermore, 51.3\% and $61.5 \%$ of the AIDA-1 and paa-positive isolates contained fimbrial adhesin genes. Among the AIDA-1-positive isolates, 48.7\% (19 of 39) were F18, which was higher than F4 (2.6\%, 1 of 39). Finally, 23.1\% (6 of 26) of the paa-positive isolates were F4 and 38.5\% (10 of 26) were F18-positive.

\section{Hemolysis and pathotype}

The relationship between hemolytic activity and pathotype is showed in Table 4. Among the pathotypes, ETEC (67.8\%, 80 isolates) was the most predominant, followed by STEC $(23.7 \%, 28$ isolates). However, just $1(0.8 \%)$ and $9(7.6 \%)$ isolates were determined as ETEC/STEC and ETEC/ STEC/enteropathogenic E. coli (EPEC), respectively. Furthermore, 85.7\% (24 of 28) of STEC and all of ETEC/STEC and ETEC/STEC/EPEC showed hemolytic activity. However, just 48.8\% (39 of 80) of ETEC showed hemolytic activity.

\section{Antimicrobial resistance}

The results of antimicrobial susceptibility testing of the pathogenic $E$. coli isolates from Korean diarrheic pigs in 2016-2017 are shown in Table 5. We confirmed high resistance rates to chloramphenicol (104 isolates, 88.1\%), tetracycline (102 isolates, 86.4\%), streptomycin (102 isolates, 86.4\%), and ampicillin (102 isolates, $86.4 \%$ ). On the other hand, some strains showed low resistance to colistin (7 isolates, 5.9\%), amoxicillin/clavulanic acid (6 isolates, 5.1\%), cefepime (3 isolates, 2.5\%), and cefoxitin (4 isolates, $3.4 \%$ ), respectively. Isolates showed moderate antimicrobial resistance to nalidixic acid (73 isolates, $61.9 \%)$, and trimethoprim/sulfamethoxazole (67 isolates, $56.8 \%)$.

A difference was observed in the antimicrobial resistance of the subclass aminoglycosides and cephalosporin I. Isolates showed high resistance to streptomycin (102 isolates, $86.4 \%)$ than gentamicin (43 isolates, 36.4\%) and neomycin (59 isolates, 50.0\%). Similar to aminoglycosides, the resistance to cephalothin (76 isolates, $64.4 \%$ ) was found to be higher than that of cefazolin (12 isolates, $10.2 \%)$.

\section{Multi-drug resistance}

Table 6 shows 118 pathogenic $E$. coli isolates with multi-drug resistance. Only one isolate (0.8\%) showed no resistance to all subclasses of the tested antimicrobials, and 90.7\% (107 isolates) showed multi-drug resistance. For the 2016-2017 isolates, 28.8\% showed a pattern of resistance to 5 antimicrobial subclasses. Furthermore, 20.3\% (24 isolates) and 26.3\% (31 isolates) were resistant to 3 and 4 antimicrobial subclasses. No isolates were found to be resistant to more than 9 antimicrobial subclasses.

Table 4. Relationship between hemolytic activity and pathotypes in 118 Escherichia coli isolates obtained from pigs with diarrhea in Korea

\begin{tabular}{lccc}
\hline \multirow{2}{*}{ Pathotypes } & \multicolumn{3}{c}{ Hemolytic activity (\%) } \\
\cline { 2 - 4 } & Hemolysis & Non-hemolysis & Total \\
\hline ETEC & $39(48.8)$ & $41(51.3)$ & $80(67.8)$ \\
STEC & $24(85.7)$ & $4(14.3)$ & $28(23.7)$ \\
ETEC/STEC & $1(100.0)$ & $0-$ & $1(0.9)$ \\
ETEC/STEC/EPEC & $9(100.0)$ & $0-$ & $9(7.6)$ \\
\hline Total & $73(61.9)$ & $45(38.1)$ & $118(100.0)$ \\
\hline
\end{tabular}

Data are expressed as number of hemolytic isolates (\% for each pathotype).

$\mathrm{ETEC}$, enterotoxigenic E. coli; STEC, Shiga-toxin producing E. coli; EPEC, enteropathogenic E. coli. 
Table 5. Antimicrobial resistance of 118 Escherichia coli isolates obtained from pigs with diarrhea in Korea

\begin{tabular}{lcc}
\hline Antimicrobial agents & No. of resistant isolates & Antimicrobial resistance (\%) \\
\hline Gentamicin & 43 & 36.4 \\
Streptomycin & 102 & 86.4 \\
Neomycin & 59 & 50.0 \\
Cephalothin & 76 & 64.4 \\
Cefazolin & 12 & 10.2 \\
Cefepime & 3 & 2.5 \\
Cefoxitin & 4 & 3.4 \\
Nalidixic acid & 73 & 61.9 \\
Ciprofloxacin & 31 & 26.3 \\
Norfloxacin & 29 & 24.6 \\
Ampicillin & 102 & 86.4 \\
AMC & 6 & 5.1 \\
SXT & 67 & 56.8 \\
Chloramphenicol & 104 & 88.1 \\
Colistin & 7 & 5.9 \\
Tetracycline & 102 & 86.4 \\
\hline Total isolates & 118 & 100.0 \\
\hline AMC, amoxicilin / clavulanic acid; SXT, trimethoprim / sulfamethoxazole. &
\end{tabular}

Table 6. Multiple resistance of 118 Escherichia coli isolates obtained from pigs with diarrhea in Korea

\begin{tabular}{lcc}
\hline \multicolumn{1}{c}{ No. of resistance ${ }^{1)}$} & No. of resistant isolates & Antimicrobial resistance (\%) \\
\hline 0 subclass & 1 & 0.8 \\
1 subclass & 2 & 1.7 \\
2 subclasses & 8 & 6.8 \\
3 subclasses & 24 & 20.3 \\
4 subclasses & 31 & 26.3 \\
5 subclasses & 34 & 28.8 \\
6 subclasses & 12 & 10.2 \\
7 subclasses & 4 & 3.4 \\
8 subclasses & 2 & 1.7 \\
\hline Multi-resistant & 107 & 90.7 \\
$(\geq 3$ subclasses & & 100.0 \\
\hline Total isolates & 118 & \\
\hline${ }^{1}$ Antimicrobial subclasses defined by the Clinical and Laborary Standards Institute are used.
\end{tabular}

\section{DISCUSSION}

Enteric colibacillosis is one of the most critical diseases in the pig industry, causing significant economic damage across the world. As such, it is very important to establish measures for the prevention of this disease, such as vaccine development or the efficient use of medicine. Not only for the development of vaccine, but also to establish efficient treatment and prevention strategies for colibacillosis, a thorough investigation of the distribution of virulence factors is needed. For this, it is important to analyze the antimicrobial resistance of the causative agents [14]. We obtained 118 pathogenic E. coli isolates from Korean diarrheic pigs during 2016-2017 and analyzed for their an- 
timicrobial resistance and virulence factors.

E. coli has many virulence factors of various types, where the virotypes are prone to changing depending on the region and period [1]. F6 was the most predominant adhesin in Korean pigs in the late 1990s, changing to F5 in the mid-2000s [15-17]. However, our results showed that the most predominant fimbria detected in Korea (i.e., for the period 2016-2017) was F18. These shifts in the predominant fimbria are believed to result from the use of autovaccines or commercial vaccines [2]. Furthermore, virotypes without fimbrial genes have recently emerged (44 isolates, 37.3\%) in Korean diarrheic pigs. In Korea, inactivated vaccines targeting F4 and F18 are used nationwide [18]. The use of these vaccines could cause antigenic variations and result in the prevalence of adhesins besides F4 and F18 in pigs.

In our study of fimbrial and enterotoxin genes, most of the isolates (98.3\%, 116 of 118) contained at least more than one enterotoxin gene. STb:EAST-1 (22 isolates, 18.6\%), which was found to not contain any fimbria, was most recently determined to be the predominant isolate in Korea. Ngeleka et al. reported that STb:EAST-1 has pathogenic characteristics in pigs and could be the cause of diarrhea [19]. F18:Stx2e (15 isolates, 12.7\%) was the second most predominant virotype in Korea in 2016-2017. Unlike other enterotoxin genes, such as LT, STa, and STb, Stx2e damages the vessel and cause edema disease with a high mortality in pigs [20]. Among fimbriae, F18 is known to be associated with edema disease. Therefore, since F18:Stx2e is the predominantly detected virotype, Korean pigs have a higher probability of developing edema disease.

Only 2 of 23 isolates (8.7\%) which showed the virotype STb:EAST-1 had hemolytic activity. The isolates with the virotype STb:EAST-1 showed a high resistance to tetracycline (22 of 23 isolates, $95.7 \%$ ), ampicillin (21 of 23 isolates, 91.3\%), and streptomycin (19 of 23 isolates, 82.6\%). However, those were all susceptible to cefazolin, cefepime, cefoxitin, and amoxicillin/clavulanic acid (data not shown). All isolates with the virotype F18:Stx2e (the second most predominant virotype in Korea in 2016-2017) showed hemolysis. Furthermore, they also had a high resistance to tetracycline (14 of 15 isolates, 93.3\%), cephalothin (13 of 15 isolates, 86.7\%), and streptomycin (12 of 15 isolates, 80.0\%). On the other hand, these isolates were all susceptible to cefoxitin, and had a low resistance to ciprofloxacin and amoxicillin/clavulanic acid (just one out of 15 isolates). Differences were found in terms of the antimicrobial resistance depending on virotype. STb:EAST-1 showed a lower resistance to gentamicin (2.7\%), trimethoprim/sulfamethoxazole (43.5\%) and colistin (4.5\%) than F18:Stx2e, which showed 26.7\%, 73.3\%, and 13.3\% resistance, respectively (data not shown). As such, the prevention strategies for and treatment of pathogenic $E$. coli should be established based on the virotype of the isolates.

We found that AIDA-1 (33.1\%, 39 of 118) was common in Korean diarrheic pigs, and AIDA-1-positive E. coli was highly related to F18 (48.7\%, 19 of 39). Niewerth et al. [21], Zhang et al. [22], and Zhao et al. [23] also reported an association between AIDA-1 and F18. It is estimated that AIDA-1 has the ability to cause cross-contamination between pigs and humans [23], such that careful observation of AIDA-1 in Korean diarrheic pigs is needed.

Unlike AIDA-1, according to Zhang et al. [22] and Byun et al. [2], paa is known to have a high association with $\mathrm{F} 4$. We confirmed that $23.1 \%$ of paa-positive $E$. coli contained the F4 gene. Although the specific role of the paa gene in the development of pathogenic $E$. coli has not yet been clearly defined, various virotypes may also appear due to horizontal gene transferability of the paa gene [24].

The eae gene plays an important role in the development of intimate adhering, as well as attaching and effacing lesions in the swine intestinal mucous membrane. It is also known to be detected in enteropathogenic E. coli (EPEC) [25]. In this study, only 1 eae-positive isolate was found, and few cases of diarrhea caused by EPEC were found in the Korean pigs. 
Hemolysis has long been considered to be one of the virulence factors of $E$. coli [26]. Here, 85.7\% (24 of 28) of STEC and all of ETEC/STEC and ETEC/STEC/EPEC were found to be highly related with hemolysis. On the other hand, only $48.8 \%$ of ETEC showed hemolytic activity. Hemolysis has been considered as an auxiliary criterion for the identification of ETEC in the clinical laboratory [26]. Here, we found that only $61.9 \%$ (73 of 118) showed hemolytic activity. Furthermore, ETEC showed no close relationship with hemolysis. Therefore, hemolysis alone is not enough to judge whether $E$. coli is pathogenic. Moreover, it is thought that the detection of pathogenic $E$. coli is necessary to search for pathogenic genes via PCR.

Recently isolated pathogenic $E$. coli from Korean diarrheic pigs showed high resistance to chloramphenicol (88.1\%), tetracycline (86.4\%), ampicillin (86.4\%), and streptomycin (86.4\%), which were similar to the monitoring results published by Denmark [6], Canada [8], and Japan [7]. According to the comparison of resistance rates by antimicrobials, tetracycline, chloramphenicol, ampicillin, and streptomycin, which have been used in large quantities for a long time in Korea, showed higher resistance rates than other antimicrobials. Similar results have been reported in other Korean reports. Cho et al. [27] reported that the rate of resistance to tetracycline was highest (97.8\%), followed by ampicillin (89.1\%). Lim et al. [10] also reported a high resistance rate of $E$. coli to tetracycline (76.1\%), ampicillin (64.6\%), and streptomycin (58.4\%). However, the resistance rates reported in this study were higher compared to those of Lim et al. [10]. This may be caused by differences in the origin of the isolates. According to results of the Korean national antimicrobial resistance monitoring systems, pathogenic bacteria tend to be more resistant to antimicrobials than bacteria isolated from normal livestock [5]. In the study by Lim et al. [10], the resistance rates of $E$. coli from normal livestock was analyzed, whereas, we tested the antimicrobial resistance of $E$. coli isolated from pigs with diarrhea.

We confirmed a very high frequency of multi-drug resistance $(90.7 \%, 107$ of 118$)$. It is difficult to directly compare multi-drug resistance rates due to the different types of antimicrobials tested, in comparison to the multi-drug resistance rates (51.7\%) of pigs with E. coli of US origin, the multidrug resistance rates of Korean pigs (90.7\%) was very high [13]. Since the regulation on the use of antimicrobials is not strict in Korea, compared to other developed countries, it is considered that the use of antimicrobials by non-specialists, such as livestock workers, and not veterinarians, may be the cause of this phenomenon [28]. Previously, we analyzed the antimicrobial resistance of $E$. coli in Korea during 2007-2016. We found that 32.6\% of $E$. coli were found to be resistant to more than 9 antimicrobial subclasses [29]. However, in this study, no isolates were found to be resistant to more than 9 antimicrobial subclasses. This indicates that there was slow tendency to decreasing antimicrobial resistance because all of antimicrobial growth promoters in feed were banned entirely in July 2011 in Korea to manage the rise in antimicrobial resistance, followed by the introduction of veterinarian prescriptions in August 2013.

We analyzed recent trends in the virulence factors and antimicrobial resistance of $E$. coli isolated from Korean diarrheic pigs and confirmed that the most dominant virotype in Korea was F18:Stx2e and STb:EAST-1. Although the antimicrobial resistance rates were found to tend to decrease compared to past studies, our findings show that these rates remain high compared to those of other developed countries. These results provide data that can be used for the prevention and treatment of enteric colibacillosis, as well as important data for the development of strategies for vaccines, antimicrobial resistance research, and use management.

\section{REFERENCES}

1. Fairbrother JM, Gyles CL. Colibacillosis. In Straw BE, Zimmerman JJ, D’ Allaire S, Taylor DJ, 
editors. Diseases of swine. 10th ed. New York, NY: John Wiley Sons; 2012. p. 723-49.

2. Byun JW, Jung BY, Kim HY, Fairbrother JM, Lee MH, Lee WK. O-serogroups, virulence genes of pathogenic Escherichia coli and pulsed-field gel electrophoresis (PFGE) patterns of O149 isolates from diarrhoeic piglets in Korea. Vet Med (Praha). 2013;58:468-76.

3. Duan Q Yao F, Zhu G. Major virulence factors of enterotoxigenic Escherichia coli in pigs. Ann Microbiol. 2012;62:7-14.

4. National Veterinary Research \& Quarantine Service. Establishment of control system of antibiotics for livestocks. Cheongju: Ministry of Food and Drug Safety; 2006.

5. Animal and Plant Quarantine Agency. Establishment of antimicrobial resistance surveillance system for livestock 2012. Sejong: Ministry of Agriculture, Food and Rural Affairs; 2013.

6. National Food Institute, Technical University of Denmark and Statens Serum Institut. DANMAP 2013 - Use of antimicrobial agents and occurrence of antimicrobial resistance in bacteria from food animals, food and humans in Denmark. Copenhagen, Denmark: DANMAP-Danish Integrated Antimicrobial Resistance Monitoring and Research Programme; 2013.

7. JVARM [Japanese Veterinary Antimicrobial Resistance Monitoring System]. Report on the Japanese veterinary antimicrobials resistance monitoring system 2012 to 2013. Tokyo, Japan: National Veterinary Assay Laboratory, Ministry of Agriculture, Forestry and Fisheries; 2016.

8. Government of Canada. Canadian integrated program for antimicrobial resistance surveillance (CIPARS) 2014 Annual Report. Guelph, ON: Public Health Agency of Canada; 2016.

9. Do KH, Byun JW, Lee WK. Prevalence of O-serogroups, virulence genes, and F18 antigenic variants in Escherichia coli isolated from weaned piglets with diarrhea in Korea during 20082016. J Vet Sci. 2019;20:43-50.

10. Lim SK, Nam HM, Moon DC, Jang GC, Jung SC. Antimicrobial resistance of Escherichia coli isolated from healthy animals during 2010-2012. Korean J Vet Res. 2014;54:131-7.

11. Bauer AW, Kirby WM, Sherris JC, Turck M. Antibiotic susceptibility testing by a standardized single disk method. Am J Clin Pathol. 1966;45:493-6.

12. Clinical \& Laboratory Standards Institute. M100-S24: Performance standards for antimicrobial susceptibility testing; twenty-fourth informational supplement. Wayne, PA: Clinical \& Laboratory Standards Institute; 2014.

13. Magiorakos AP, Srinivasan A, Carey RB, Carmeli Y, Falagas ME, Giske CG, et al. Multidrug-resistant, extensively drug-resistant and pandrug-resistant bacteria: an international expert proposal for interim standard definitions for acquired resistance. Clin Microbiol Infect. 2012;18:268-81.

14. Fairbrother JM, Nadeau E, Gyles CL. Escherichia coli in postweaning diarrhea in pigs: an update on bacterial types, pathogenesis, and prevention strategies. Anim Health Res Rev. 2005;6:17-39.

15. Kwon D, Choi C, Jung T, Chung HK, Kim JP, Bae SS, et al. Genotypic prevalence of the fimbrial adhesins (F4, F5, F6, F41 and F18) and toxins (LT, STa, STb and Sbc2e) in Escherichia coli isolated from postweaning pigs with diarrhoea or oedema disease in Korea. Vet Rec. 2002;150:35-7.

16. Kwon D, Kim O, Chae C. Prevalence of genotypes for fimbriae and enterotoxins and of $\mathrm{O}$ serogroups in Escherichia coli isolated from diarrheic piglets in Korea. J Vet Diagn Invest. 1999;11:146-51.

17. Lee SI, Rayamahji N, Lee WJ, Cha SB, Shin MK, Roh YM, et al. Genotypes, antibiogram, and pulsed-field gel electrophoresis profiles of Escherichia coli strains from piglets in Korea. J Vet Diagn Invest. 2009;21:510-6.

18. Chae MJ, Cho JK, Lee YJ. Virulence genes of Escherichia coli isolates from piglets with diar- 
rhea in Korea.J Anim Vet Adv. 2012;11:9-12.

19. Ngeleka M, Pritchard J, Appleyard G, Middleton DM, Fairbrother JM. Isolation and association of Escherichia coli AIDA-I/STb, rather than EAST1 pathotype, with diarrhea in piglets and antibiotic sensitivity of isolates.J Vet Diagn Invest. 2003;15:242-52.

20. Nguyen UV, Coddens A, Melkebeek V, Devriendt B, Goetstouwers T, Van Poucke M, et al. High susceptibility prevalence for F4+ and F18+ Escherichia coli in Flemish pigs. Vet Microbiol. 2017;202:52-7.

21. Niewerth U, Frey A, Voss T, Bouguénec CL, Baljer G, Franke S, et al. The AIDA autotransporter system is associated with F18 and Stx2e in Escherichia coli isolates from pigs diagnosed with edema disease and postweaning diarrhea. Clin Diagn Lab Immunol. 2001;8:143-9.

22. Zhang W, Zhao M, Ruesch L, Omot A, Francis D. Prevalence of virulence genes in Escherichia coli strains recently isolated from young pigs with diarrhea in the US. Vet Microbiol. 2007;123:145-52.

23. Zhao L, Chen X, Xu X, Song G, Liu X. Analysis of the AIDA-I gene sequence and prevalence in Escherichia coli isolates from pigs with post-weaning diarrhoea and oedema disease. Vet J. 2009;180:124-9.

24. Leclerc S, Boerlin P, Gyles C, Dubreuil JD, Mourez M, Fairbrother JM, et al. Paa, originally identified in attaching and effacing Escherichia coli, is also associated with enterotoxigenic E. coli. Res Microbiol. 2007;158:97-104.

25. Alonso CA, Mora A, Díaz D, Blanco M, González-Barrio D, Ruiz-Fons F, et al. Occurrence and characterization of stx and/or eae-positive Escherichia coli isolated from wildlife, including a typical EPEC strain from a wild boar. Vet Microbiol. 2017;207:69-73.

26. Sato JPH, Takeuti KL, Andrade MR, Koerich PKV, Tagliari V, Bernardi ML, et al. Virulence profiles of enterotoxigenic Escherichia coli isolated from piglets with post-weaning diarrhea and classification according to fecal consistency. Pesq Vet Bras. 2016;36:253-7.

27. Cho JK, Ha JS, Kim KS. Antimicrobial drug resistance of Escherichia coli isolated from cattle, swine and chicken. Korean J Vet Public Health. 2006;30:9-18.

28. Do KH, Byun JW, Lee WK. Serogroups, virulence genes and antimicrobial resistance of F4+ and F18+ Escherichia coli isolated from weaned piglets. Pak Vet J. 2019;39:266-270.

29. Do KH, Byun JW, Lee WK. Antimicrobial resistance of Stx2e positive Escherichia coli before and after ban on antibiotic growth promoters. J Biomed Transl Res. 2017;18:84-92. 